\title{
The Application of Big Data in the "Internet Plus" Era Lin Shi
}

Tianjin Maritime College, Tianjin, China, 300350

Keywords: Internet plus; Data processing; Data value; Cloud computing

\begin{abstract}
Since the beginning of 2015, "Internet plus" has become China's important strategy of national development, promote the integration of industrialization and informatization. In the "new model Internet plus", society, economy, industry, service industry and other areas of information showing the rapid increase trend, Internet carrying value data, and big data analysis, mining and processing has become the mainstream of information technology. This paper mainly discusses the application of big data "Internet plus" background processing technology, challenges and prospects of big data analysis.
\end{abstract}

\section{大数据处理在 “互联网 + ” 时代中的应用}

\author{
石琳 \\ 天津海运职业学院 中国 天津市 300350
}

摘要: 自 2015 年始, “互联网+”已经成为我国国家发展重要战略之一, 促进工业化和信息化 的高度融合。在 “互联网+”的新型模式影响下, 社会、经济、工业、服务业等范畴的信息呈 现迅猛增加趋势, 互联网承载着大量的价值数据, 而大数据的剖析、挖掘及处理已成为主流 信息技术。本文主要探讨 “互联网+” 背景下的大数据处理技术的应用, 分析大数据带来的挑 战和展望。

\section{关键词：互联网+；大数据处理；价值数据；云计算}

\section{1. 引言}

当今社会, “互联网+”已成为人们熟悉的不能再熟悉的标志性词语。2012 年 “互联网+”一 词被互联网业界初次提出, 从而进一步表明了 2007 年提出的 “互联网化” 理念, 旨在互联网 与传统行业的高度交融。第十二届全国人民代表大会第三次会议于 2015 年 3 月 5 日召开, 会 上李克强总理将 “互联网+” 这一战略嵌入到中国经济发展整体规划中, 强调在推进移动互联 网、云计算、大数据、物联网等信息技术与传统行业相融会的同时, 带动电子商务、工业互 联网和互联网金融等行业的蓬勃发展, 并努力拓展国际互联网市场, 进而打造新型现代化行 业增长点。

\section{2. “互联网+” 时代}

\section{1. “互联网+” 的现状}

“互联网+”即 “互联网+传统行业”，在信息技术和互联网的背景下，融会各领域的传统行业 和互联网合作, 在全新的范畴中, 缔造全新社会生产力和生态环境, 不断推进经济社会发展。

“互联网+”时代的到来，打破了许多行业间的界限和代沟，信息资源在互联网上以增量、快 速、多样等方式传输。传统行业对自身的产业发展分析和看待问题的方式, 已经不再局限于 某一角度和方向。“互联网+” 行业已渐渐进入人们的生活。除了像互联网+工业、互联网+金 融、互联网+教育等第三产业外, “互联网+” 正逐渐向着第一、二产业渗透, 如互联网能源、 互联网外贸等。而大数据在 “互联网+” 行业中扮演着相当必要的角色, 大数据服务、大数据 
营销、大数据金融等模式, 都将推动着 “互联网 + ” 的发展历程, 增进互联网与各行业间的融 会成长。

\section{2. “互联网+” 与大数据}

大数据处理技术对 “互联网+”的时代历程起着举足轻重的推进作用。如淘宝等电子商务平台, 如何利用大数据技术分析统计，帮助消费者对上牌的价值深入了解; 如何使消费者在大量的 货品中挑选出自己满意的商品, 这些都会是未来大数据技术发展的推动力。

电商平台是基于群体性行为的分析篮选过程, 依赖于大数据处理技术。通过收集、分析出用 户汶览、关注等大量无序数据, 以及对各项数据的比对、分析的应用。所以, 大数据处理技 术加快了传统行业向 “互联网+”现代经营模式变革的步伐。

大数据处理技术在企业的产品优化创新和用户体验方面, 带来了全新模式和先进理念。随着 大数据技术的引入，在原有的评价体系的基础上，进一步深入和拓展，打破信息防御，增强 互联网平台上的用户、商家和服务等数据信息的可信度和透明度。随着 “互联网+” 时代下的 大数据的不断深化，必将形成 “大数据 + ” 时代，使 “加” 入互联网行业的信息化和数据化程 度发展到一个全新的高度和领域, 即 “大数据 + ”，深化 “+” 入互联网的行业信息化和数据化。 “大数据+” 不单融会着数据化的网络产业, 还将不同关系信息网中 “互联网+” 模式缔造为 融会世界的新纽带, 从而创建一个新的 “中国+” 时代。

\section{3. 大数据处理技术}

\section{1. “大数据” 的定义及特征}

“大数据” 是在规定时间内无法应用常用软硬件等工具去感知、获取、操作和处理的大量数 据的集合。大数据的范围约在 $10 \mathrm{~TB}$ 至 $1 \mathrm{~PB}$ 以上, 除了关注于数据量的大小, 还应着重数据 的种类和处理的速度等方面要素。现代化处理模式下的大数据, 具备较强的决策能力、洞察 能力, 具备多样化, 且较高增长率的海量信息资产。大数据一般涉及 2 种以上（含 2 种）的 数据形式, 收集超过 $100 \mathrm{~TB}$ 的高速、实时数据流, 它超过了任何一个计算机处理能力的庞大 数据量。

大数据从早期的 $3 \mathrm{~V}$ (Value)、 $4 \mathrm{~V}$ 到现在的 $5 \mathrm{~V}$ ，其中包含 5 个特征。

$>$ Volume (大体量): 数据体量巨大, 收集和分析的数据量非常大, 可从数百 $\mathrm{TB}$ 到数十 $\mathrm{PB}$ 、甚至 $\mathrm{EB}$ 的规模。

$>$ Velocity (时效性): 处理速度快, 大数据需要在规定时间内实时进行数据的分析、决策 和处理。

$>$ Variety (多样性): 数据类别大, 大数据中有各种类型的数据, 如结构化、半结构化和非 结构化等多种数据形态。

$>$ Veracity (准确性): 数据真实性, 大数据来源于真实的数据环境, 从而保证从海量网络 数据库中获取数据的准确性和真实性, 能够诠释和还原出现实事件的整个进程。

$>$ Value (大价值): 价值密度低, 商业价值高, 大数据包含很多深度的价值, 大数据分析、 挖掘和利用可抓住机遇, 收获价值。

\section{2. 大数据技术框架}

大数据处理技术开创了计算机运行的全新模式，可以处理不同来源的各类海量数据，其关键 技术是对各类海量数据的存储和分析。传统数据的收集来源较单一, 需要分析处理的数据量 相应比较小，常用的关系型数据库或并行数据库就能完成处理操作。自从 “大数据” 的引入 以来, 相应的不同来源的数据呈现多样化, 需要存储和分析越来越庞大、复杂的海量数据, 且数据处理质量的要求越来越高, 侧重于大数据处理的可靠性和高效性, 如此就需要并行计 算能力来提高数据处理速率。大数据是一个庞大且复杂的信息资源库, 大数据处理技术就是 对数据信息的获取、传输、处理的过程, 它会直接处理这些海量的结构化、半结构化和非结 构化数据，并分析出预测的结果，其过程即为 “大数据处理技术”。 


\section{3. 大数据处理技术}

近年来, 大数据处理技术工具层出不穷, 从最早的 Hadoop 开源平台, 到现在 S4、Storm 等 大数据处理工具，很多公司都拥有针对自己特定用途的处理平台。

\section{3.1 Hadoop}

但凡谈到大数据, 必然会提到 Hadoop, 短短几年时间, Apache Hadoop 已成长为适用于非结 构化数据的首选大数据分析解决方案。Hadoop 是目前最为流行的大数据处理技术, 由 Apache 公司为实现 Google 的 MapReduce 编程模型的云计算平台, 具有高效的可伸缩性, 能够处理 PB 级数据, 很多大数据处理工具都是在基础上改进而成, Hadoop 已逐步成为大数据处理工 具的标准。其由 Java 语言编写, Linux 操作系统环境下运行，包括分布式文件系统 (HDFS)、 数据处理（MapReduce）、数据库（HBase、Cassandra）、数据仓库（Hive）等模块。

HDFS 集群结构包括 Namenode、Datanode、Client 三类节点, 其间通信均在 TCP/IP 下进行。 首先 Client 发送命令请求, 管理命令空间信息的 Namenode 接收命令请求, 并分配给 Datanode 们处理计算, 随后各个 Datanode 节点将处理结果信息反馈给 Namenode, 以确认 Datanode 节 点的当前状态，同时反馈节点信息给 Client，并写入数据节点存储。

MapReduce 中有 Map 和 Reduce 两个并行处理环节。首先将输入的原始数据分区分片，随之 分配给各 Map 任务进行处理操作, 并对经由 Map 函数处理过的过程结果 Key-Value 集合输 出, 并经过简单压缩合并后, 拷贝给 Reduce 任务, 以降低 Reduce 处理的任务量, 提高其工 作效率。Reduce 将接收到各 Map 任务处理的结果进行整合分析，形成最终结果输出。

\section{3.2 Storm}

Storm 是 Twitter 开源的高容错性的、分布式实时的计算机系统。相对于 Hadoop 用于批处理, Storm 则用于实时处理, 它可以实时地处理数据, 并保证处理数据的完整、全面。Storm 为了 转换没有终端的数据流, 创建了拓扑结构, 且持续处理随时出现的数据。Storm 集群结构主 要由主节点和工作节点两部分所构成。主节点上运行的守护进程 Nimbus，执行分配任务和监 测故障的命令。而工作节点群上运行的守护进程 Supervisor, 需要实时监听分配任务的机器, 并监控 Nimbus 所下达命令的工作进程的启动和关闭状况。

\section{3.3 Spark}

Spark 开源计算系统相似于 Hadoop, 但又不同于 Hadoop, 差异之处更突出了 Spark 的某些工 作负载能力的优越性。Spark 的内存分布数据集不仅可以进行交互式查询, 还能优化迭代工作 的负载。Spark 是用 Scala 语言编写的分布式内存计算框架, Spark 和 Scala 能够紧密集成, 其 中的 Scala 可以像操作本地集合对象一样轻松地操作分布式数据集。其核心抽象模型是弹性 分布式数据集 (RDD), 且创建了分布式 API, 可直接处理数据集, Spark 提升了一到两个数 据级，大大提高了其数据处理效率。Spark 上的软件栈 BDAS 包含着若干个 Spark 处理工具。

\section{4. 大数据发展趋势}

随着大数据的不断发展和研究，其巨大价值在被不断挖掘的过程中，大数据将不断促进传功 产业向电商产业转型的速度，同时影响着现代信息技术的发展趋势。

众所周知，大数据时代给人们带来了巨大的数据信息资源，使数据资源化成为当今社会关注 焦点, 也成为企业发展的重要战略目标。大数据资源不仅成为了企业资产的标志，更是提高 了企业竞争力。

如果说大数据是原始资源的累积，则云计算就是一把顺手的钢刀，二者相辅相成。云处理是 为大数据而生, 它能提供大数据处理所需要的一切基础设备, 是提炼大数据的重要平台, 大 数据处理技术与云计算技术高度融合。此外，通过云平台引入了一系列新兴技术形态，如物 联网、移动互联网等都逐步成为大数据处理数据的助力, 从而更激发了大数据时代的发展进 程。

随着大数据的爆炸式发展，人们开始慢慢着眼于大数据安全问题。大数据带来了存储数据的 
意识革命, 海量原始数据的逐步增长, 数据存储的安全性面临着巨大风险和挑战, 如何从现 代化技术层面提升网络安全、保护隐私数据一直是社会关注的重大问题, 也是制约大数据发 展的重要环节。

\section{5. 结束语}

“互联网+” 环境下的大数据时代已经到来，随时带来了巨大机遇，同时也带来了一系列技术 难题。本文从大数据处理技术角度阐析, 探讨 “互联网 + ” 时期下的 “大数据” 进程, 以及近 年来大数据所面临的机遇与挑战, 如何从大数据中发现创新价值, 如何提高大数据处理的实 时性，以及大数据环境下的信息安全等问题仍是当前亟待解决的问题。

\section{参考文献}

[1] 方巍. 大数据: 概念、技术及应用研究综述 [A].南京信息工程大学学报, 2014, 6(5): 405-419.

[2] 冯登国，张敏，李昊.大数据安全与隐私保护[J].计算机学报, 2014, 37(1): 246-258.

[3] 刘峰.互联网进化论[M].北京：清华大学出版社, 2012.

[4] 李国杰.大数据研究的科学价值[J].中国计算机学会通讯, 2012, 8(9): 8-15.

[5] 徐子伟, 张陈斌, 陈宗海. 大数据技术概述 $[\mathrm{A}]$. 系统仿真技术及其应用学术论文集 $[\mathrm{C}], 2014$, 15: 403-409.

[6] 张引, 陈敏, 廖小飞. 大数据应用的现状与展望 [J]. 计算机研究与发展. 2013, 50（2): 216-233.

[7] 孟小峰, 慈祥.大数据管理: 概念、技术与挑战 [J]. 计算机研究与发展, 2013, 50(1): 146-169.

[8] 贾元昕, 杨明川, 孙静博. 大数据在“互联网+”进程中的应用 $[\mathrm{J}]$. 电信技术, 2015, 6: 14-20.

[9] 孙玲玲.浅谈“互联网+”[A].第二十九届中国（天津）IT、网络、信息技术、电子、仪器仪 表创新学术会议[C],2015,9:144-147.

[10] 维克托. 迈尔一舍恩伯格, 肯尼思. 库克耶.大数据时代 $[\mathrm{M}]$. 杭州: 浙江人民出版社, 2013.

\section{References}

[1] Fang Wei. Big data: Conceptions, key technologies and application [A]. Journal of Nanjing University of Information Science and Technology, 2014, 6(5): 405-419.

[2] Feng Dengguo, Zhang Min, Li Hao. Big Data security and privacy protection [J]. Chinese Journal of Computers, 2014, 37 (1):246-258.

[3] Liu feng. Internet evolution theory [M]. Bei Jing: Tsinghua university press, 2012.

[4] Li Guojie. Scientific value on Big Data research [J]. Communications of China Computer Federation, 2012, 8(9):8-15.

[5] Xu Ziwei, Zhang Chenbin, Chen Zonghai. Overview of big data technology [A]. System simulation technology and its application[C], 2014, 15: 403-409.

[6] Zhang Yin, Chen Min, Liao Xiaofei. Big data applications: A survey [J]. Computer research and development, 2013, 50(2):216-233.

[7] Meng Xiaofeng, Ci Xiang. Big Data management: Concepts, techniques and challenges [J]. Journal of Computer Research and Development, 2013, 50(1): 146-169. 
[8] Jia Yuanxin, Yang Mingchuan, Sun Jingbo.The application of big data in the "Internet plus" in the process of $[\mathrm{J}]$. Telecommunications Technology, 2015, 6:14-20.

[9] Sun Lingling. Discussion on "Internet plus"[A].The 2015 China (Tianjin) twenty-ninth 'IT, network, information technology, electronics, instrumentation innovation Academic Conference [C], 2015, 9:144-147.

[10] Victor-Maier-Schoenberg, Kenneth Kukye. Big data Era [M].Hang Zhou: Zhejiang people's Publishing House, 2013.

作者简介: 石琳 $(1981-)$, 女, 天津市人, 副教授, 主要研究计算机程序设计及网络构建 方向。 